\title{
Cerebrovascular Risk Factors, Vascular Disease, and Neuropsychological Outcomes in Adults With Major Depression
}

\author{
Patrick J. Smith, BA, James A. Blumenthal, PhD, Michael A. Babyak, PHD, Benson M. \\ HOffman, PhD, P. Murali doraiswamy, MD, Robert Waugh, MD, Alan Hinderliter, MD, and \\ Andrew Sherwood, PhD \\ Department of Psychiatry and Behavioral Sciences (P.J.S., J.A.B., M.A.B., B.M.H., P.M.D., R.W., \\ A.S.), Duke University, Durham, North Carolina; Department of Medicine (A.H.), University of \\ North Carolina at Chapel Hill, North Carolina
}

\begin{abstract}
Objective-To investigate the relationship of cerebrovascular risk factors (CVRFs), endothelial function, carotid artery intima medial thickness (IMT), and neuropsychological performance in a sample of 198 middle-aged and older individuals with major depressive disorder (MDD).

Neuropsychological deficits are common among adults with MDD, particularly among those with CVRFs and potentially persons with subclinical vascular disease.
\end{abstract}

Methods-CVRFs were indexed by the Framingham Stroke Risk Profile (FSRP) and serum cholesterol levels obtained by medical history and physical examination. Patients completed a neuropsychological test battery including measures of executive functioning, working memory, and verbal recall. Vascular function was indexed by carotid artery IMT and brachial artery flow mediated dilation (FMD). Hierarchical multiple regression analyses were used to investigate the association between CVRFs, vascular disease, and neurocognitive performance.

Results-Greater FSRP scores were associated with poorer executive functioning ( $\mathrm{b}=-0.86 ; p$ $=.041)$ and working memory $(\mathrm{b}=-0.90 ; p=.024)$. Lower high-density lipoprotein levels also were associated with poorer executive functioning $(\mathrm{b}=1.03 ; p=.035)$. Higher IMT $(\mathrm{b}=-0.83 ; p$ $=.028)$ and lower FMD $(\mathrm{b}=1.29 ; p=.032)$ were associated with poorer executive functioning after controlling for CVRFs. Lower FMD was also associated with poorer working memory $(b=$ $1.58 ; p=.015)$.

Conclusions-Greater CVRFs were associated with poorer neuropsychological performance. Vascular dysfunction also was associated with neuropsychological decrements independent of traditional CVRFs.

\section{Keywords}

cerebrovascular risk; vascular disease; neuropsychological performance; depression; endothelial function

\section{INTRODUCTION}

Cerebrovascular disease is a major health burden in the United States (1) and is associated with an increased incidence of cognitive impairment (2). Previous studies have shown that greater levels of cerebrovascular risk factors (CVRFs), such as hypertension, diabetes,

Copyright $@ 2007$ by the American Psychosomatic Society

Address correspondence and reprint requests to Patrick Smith, Box 3119, Department of Psychiatry and Behavioral Sciences, Duke University Medical Center, Durham, NC 27710. Smith562@mc.duke.edu. 
smoking (3), and increased serum cholesterol levels are associated with poorer neuropsychological functioning (4) as well as increased rates of cognitive decline (5). Furthermore, a growing body of evidence indicates that neuropsychological deficits may be predictive of incident cardiovascular disease and stroke (6) as well as dementia (7) and may therefore represent a neurobehavioral index of cerebrovascular risk.

Recent studies have shown that psychosocial factors, particularly depression, may be associated with an increased risk of cerebrovascular disease (8). The relationship between CVRFs and neuropsychological performance is particularly important for individuals with major depressive disorder (MDD) because both CVRFs (9-13) and neuropsychological deficits $(14,15)$ are prevalent in this population. Furthermore, CVRFs have been associated with an increased risk for the development of mild cognitive impairment (16), a variety of neuroanatomical abnormalities (17-20), and an increased incidence of vascular depression (21). Although CVRFs have been reported to be associated with poorer cognitive performance among individuals with late-onset depression (21), the relationship between CVRFs and neuropsychological functioning among individuals with MDD without significant medical comorbidities remains unclear.

A growing body of evidence also indicates that vascular functioning may be related to neuropsychological performance $(22,23)$ and may be compromised among individuals with MDD $(24,25)$. Although CVRFs are associated with poorer vascular functioning (26), the extent to which diminished vascular functioning is associated with impairments in neuropsychological performance independent of CVRFs has not been examined. Furthermore, although individual CVRFs, such as increased systolic blood pressure, have been shown to be related to incident cerebrovascular disease (27), few studies have examined the relationship between CVRFs and neuropsychological performance in a graded, dose-response fashion $(5,28)$. Similarly, recent studies demonstrating a relationship between vascular functioning and neuropsychological performance functioning have relied on structural indicators of systemic vascular dysfunction such as carotid plaque (29) and increased carotid artery intima medial thickness (IMT) $(22,23)$, but have not examined vascular function in the context of multiple CVRFs.

\section{METHODS}

\section{Experimental Design}

The data reported in this study were obtained from a larger trial that examined the effects of exercise and antidepressant medication in the treatment of middle-aged and older individuals with MDD based on clinician-diagnosed Diagnostic and Statistics Manual 4th edition (DSM-IV) criteria (30). Exclusion criteria were as follows: a) a primary psychiatric diagnosis other than MDD; b) medical contraindications preventing participation in either the exercise or medication arm of the study; and c) current antidepressant use. Participants were recruited from October 2000 to September 2005. The study protocol was approved by a Duke Institutional Review Board.

\section{Cerebrovascular Risk Factors}

Framingham Stroke Risk Profile (FSRP)—CVRF scores were determined at the baseline screening and physical examination using the FSRP, a risk assessment tool used to assess the 10-year incidence of stroke (31). Risk factors used to assess stroke risk include systolic blood pressure, use of antihypertensive therapy, diabetes mellitus, cigarette smoking, cardiovascular disease, and atrial fibrillation $(31,32)$. Because age served as a covariate in our final analyses, it was not included in calculating FSRP scores. Furthermore, left ventricular hypertrophy, a component of the original FSRP score, was not used in the 
current analysis because this information was not routinely obtained. Participants with a prior history of stroke $(n=4)$ were excluded from the final analyses.

\section{Serum Cholesterol}

High-density lipoprotein (HDL) and low-density lipoprotein (LDL) cholesterol were measured enzymatically (LabCorp, Research Triangle Park, NC). HDL cholesterol was estimated by assay of the supernatant remaining after precipitation of serum LDL with dextran sulfate plus magnesium chloride. Participants fasted for 12 hours before this assessment.

\section{Measures of Subclinical Vascular Disease}

Intima Medial Thickness (IMT) in Carotid Arteries-Carotid artery IMT was assessed by a high-resolution B-mode ultrasound vascular imaging system (Acuson Aspen, Mountain View, CA) with a 10-Mhz linear array transducer. Ultrasound examinations of the far wall of the left and right common carotid arteries (CCAs) were used to acquire longitudinal images spanning $2 \mathrm{~cm}$ proximal to the carotid bulb. IMT of the far wall of the left and right CCAs was measured over a 1-cm segment using edge detection software (Carotid Analyzer 5.0.5, Medical Imaging Applications LLC, Iowa City, IA). Far wall measurements only were used as near wall measurements have been shown to have limited reliability (33).

\section{Flow Mediated Dilation (FMD) and Nitroglycerin Mediated Dilation (GTN-D) of} the Brachial Artery-FMD and GTN-D of the brachial artery were assessed in the morning, after overnight fasting. Participants were instructed to avoid use of vasoactive medications (a list was provided to participants) from midnight until after the FMD assessments were completed. Longitudinal B-mode ultrasound images of the brachial artery, 4 to $6 \mathrm{~cm}$ proximal to the antecubital crease, were obtained using an ultrasound platform (Aspen, Mountain View, CA) with a 10-MHz linear array transducer. Images were obtained under the following conditions: a) after 10 minutes of supine relaxation; b) during reactive hyperemia, induced after inflation for 5 minutes to suprasystolic pressure $(\sim 200 \mathrm{~mm} \mathrm{Hg})$ of a pneumatic occlusion cuff placed around the forearm; c) after an additional 10 minutes relaxation period in the supine position; and d) after the administration of $400 \mu \mathrm{g}$ sublingual glyceryl trinitrate (GTN) spray. End-diastolic images were stored to a magnetic-optical disk and arterial diameters were measured as the distance between the proximal and distal arterial wall intima media interfaces, using PC-based software (Brachial Analyzer Version 4.0, Medical Imaging Applications LLC, Iowa City, IA).

Peak hyperemic flow was assessed by Doppler velocity measurement during the first 10 seconds post deflation of the occlusion cuff, and hyperemic flow response was defined as percent change in flow relative to resting baseline. Peak FMD response was assessed from 10 to 120 seconds post deflation of the cuff, with peak arterial diameter quantified using polynomial curve fitting. FMD was defined as the maximum percent change in arterial diameter relative to resting baseline. GTN-D was defined as peak percent change in arterial diameter assessed 3 to 5 minutes after the administration of GTN.

\section{Assessment of Cognitive Functioning}

All participants underwent a standard neuropsychological test battery to assess performance in multiple domains of cognitive functioning. Tests were selected based on the brevity of their administration, availability of normative data, and the availability of multiple forms for repeat testing. The following instruments were administered individually by a trained research assistant: 
- Weschler Memory Scale Logical Memory Subset (34). The Logical Memory Subtest requires participants to repeat a story read to them aloud by the examiner. Immediate recall is evaluated using a verbatim scoring procedure. This test is used to assess discourse memory and oral language comprehension.

- Controlled Oral Word Association Test (COWAT) (35). The COWAT requires participants to generate as many words as possible beginning with different letters of the alphabet in a 1-minute period. The letters $\mathrm{C}, \mathrm{F}, \mathrm{L}$ and $\mathrm{P}, \mathrm{R}, \mathrm{W}$ were used in the current study. The COWAT is designed to assess working memory and verbal fluency.

- Wechsler Adult Intelligence Scale-Revised Digit Symbol Substitution Test (DSST) (36). The DSST is a measure of executive functioning in which participants are asked to draw symbols that match one of 10 digits copied from a key. Scores on this task are the number of correct symbols drawn in a 90-second time period.

- $\quad$ Ruff 2 and 7 Test (37). The Ruff 2 and 7 Test is a paper-and-pencil test that assesses perseveration, a component of executive functioning. Participants are required to cross out all instances of the numbers 2 and 7 under a) the first condition in which they are embedded among other digits and b) the second condition in which they are embedded among letters. Stimuli are presented in consecutive blocks of three lines (each line consisting of 10 targets and 40 distracters) representing each condition with a 15 -second time limit per block, in pseudorandom order. The score is the total number of correct cancellations within a 5-minute time period.

- Verbal Paired Associates (34). The Verbal Paired Associates Test assesses working memory by presenting participants with a list of eight word pairs, half of which had high association (e.g., baby-cries) and the remaining half which had low association (e.g., school-grocery). After the list has been read aloud, participants are presented with one word from each pair and are asked to name a corresponding word from the original list. The list of words is then repeated on two subsequent trials and the recall process is repeated. Scores of high association recall and low association recall are calculated.

- Weschler Digit Span (36). The Digit Span Test (DST) assesses attention and working memory. In this test, participants are asked to recall a list of consecutive numbers that become sequentially longer as the participants answer more trials correctly. In the first trial, participants are asked to recall the numbers in the same order in which they were presented. In the second trial, participants are asked to recall the numbers in the reverse order from which they were presented.

- Stroop Color and Word Test (38). The standardized version of the Stroop test used in the current study consists of three sections: word, color, and color-word. In each section, participants are presented with a page consisting of five columns, each with 20 items for a total of 100 items per page. Within each section, the score is the total number of words read aloud in 45 seconds. The word section requires participants to read a list of color words ("RED," "BLUE," and "GREEN") as quickly as possible. For the color section, participants name aloud the color of a series of colored bars printed on the page. The color-word list is similar to the color list in that the task is to name the color in which each item is printed. The items in this list, however, are color names, in which the names of the items differ from the colors in which they are printed (e.g., the word RED is printed in blue ink). The word and color tests are believed to measure processing speed, whereas the colorword section (also known as the "interference" section) is believed to measure selective attention, a component of executive functioning. 
- Animal Word Fluency Test (39). The Animal Fluency Test is a test of semantic fluency and working memory in which the participant is instructed to generate words from a specified category (in this case, animals). The score is the total number of animals generated in a 60 -second time period.

- Trail Making Test Part A (TMT-A) (40). The TMT-A requires participants to draw consecutive lines between the numbers 1 through 25 as fast as possible. The score is the time (in seconds) required for completion and is believed to measure processing speed and selective attention.

- Trail Making Test Part B (TMT-B) (40). The TMT-B requires participants to draw consecutive lines alternating back and forth between the numbers 1 and 13 and the letters A through $\mathrm{L}$. The score is the time (in seconds) required for completion and is believed to measure visual search and attentional processes associated with executive functioning.

\section{Assessment of Depression}

Diagnostic Interview Schedule-The presence of MDD was determined by a trained clinical psychologist using the Diagnostic Interview Schedule (41). All participants met the criteria for MDD based on DSM-IV (42).

Hamilton Rating Scale for Depression (HRSD)—Depression severity was assessed using the HRSD (43), a 17-item clinical rating scale that assesses the severity of depressive symptoms such as changes in appetite, sleep, and depressed mood.

\section{Statistical Analysis}

Data Reduction-To minimize the number of statistical tests in the present analysis, we used principle axis factor analysis to combine information from the 10 individual neuropsychological tests into three cognitive domain scores. A Scree test was used to determine the total number of factors retained for analysis. A minimum loading of 0.40 was required and Promax rotation was used. Based on these results, we created unit-weighted composite scores by standardizing the individual neuropsychological test scores and then summing all subtests relevant to a given domain. These composites were then used as the criterion variable in the regression models described below. TMT-A and TMT-B and the COWAT were log-transformed before analysis.

Data Analysis-A separate hierarchical multiple regression analysis was conducted for each cognitive domain to determine if vascular functioning was associated with neuropsychological performance independently of CVRFs. For each model, a hierarchical approach was used in which variables were entered in blocks: a) background characteristics (age, education, depression severity, and baseline arterial diameter (FMD analyses only)) and b) CVRFs (FSRP and lipids). After these blocks were entered, we entered IMT alone and then FMD alone (without IMT) to examine the contribution of each variable in the presence of the first two blocks. As a final step, IMT and FMD were entered together in addition to the first two blocks. Multiple imputation based on the parametric maximum likelihood regression was used to account for missing data. Model assumptions of additivity, linearity, and distribution of residuals were evaluated and found to be adequate before analysis. Regression coefficients were scaled using the interquartile range of the predictor variable. This scaling allows the coefficient to be interpreted as comparing a "typical" person in the middle of the upper half of the predictor distribution with a "typical" person in the middle of the lower half of the predictor distribution. 


\section{RESULTS}

\section{Sample Characteristics}

Data from 198 (98\%) of the original 202 patients were used in the final analysis, including 150 women (76\%) and 48 men (24\%). Background characteristics for the sample are presented in Table 1. The mean age for the overall sample was $51.6 \pm 7.5$ (standard deviation) years. The majority of patients were Caucasian and college-educated. Most participants were either overweight or obese and many were current or former smokers. On average, participants had mildly elevated blood pressure and approximately one quarter of the sample reported regular use of antihypertensive medications. Lipid levels were increased, but relatively few participants (8.6\%) reported regular use of lipid-lowering medications. Most participants were free from any cardiac condition with only four reporting a history of atrial fibrillation and two patients with a documented history of coronary artery disease. The CVRF score in the current sample was $5.4 \pm 3.2$, indicating participants had relatively low levels of stroke risk. Most participants were mildly to moderately depressed (Ham-D score $=16.7 \pm 4.3$ ).

\section{Neuropsychological Functioning}

Neuropsychological characteristics of the study sample are presented in Table 2. Results from the common factor analysis demonstrated three factors from the neuropsychological battery corresponding to three cognitive domains, which we have labeled executive functioning (Stroop interference, Digit Symbol Substitution, Ruff 2 and 7 Test, and Trail Making Tests), working memory (Controlled Oral Word Association Task, Animal Naming, TMT-B, and DST), and verbal recall (Weschler Logical Memory Subset and the Verbal Paired Associates Tests). The correlations between individual subtests and composite neuropsychological test scores are presented in Table 3 .

\section{Relationship Between CVRFs and Neuropsychological Functioning}

Greater FSRP scores were associated with lower executive functioning $(\mathrm{b}=-0.86 ; p=.041)$ and working memory $(\mathrm{b}=-0.90 ; p=.024)$; the association with verbal recall was smaller in magnitude and not statistically significant $(\mathrm{b}=-0.22 ; p=.283)$. Higher levels of HDL-C were associated with better executive functioning scores $(\mathrm{b}=1.03 ; p=.035)$; the association with working memory was smaller and not statistically significant $(\mathrm{b}=0.78 ; p=.119)$, whereas the association with verbal recall was small and not statistically significant $(\mathrm{b}=$ $-0.01 ; p=.948)$.

\section{Hierarchical Regression}

Results from the hierarchical regression models of executive functioning and working memory are presented in Tables 4 and 5, respectively. As shown in Table 4, the inclusion of CVRFs significantly improved the fit of the executive functioning model (adjusted $\mathrm{R}^{2}$ change $=4 \% ; p=.011$ ) and marginally improved our model of working memory (adjusted $\mathrm{R}^{2}$ change $\left.=2.1 \% ; p=.065\right)$. IMT was significantly related to executive functioning after controlling for CVRFs ( $p=.032$ ) (Figure 1), but was not significantly related to working memory $(p=.28)$. FMD also significantly improved the fit of the executive functioning (adjusted $\mathrm{R}^{2}$ change $=2.6 \% ; p=.032$ ) (Figure 2 ) and working memory (adjusted $\mathrm{R}^{2}$ change $=2.4 \% ; p=.015)$ (Figure 3 ) models. When IMT and FMD were entered simultaneously into our model of executive functioning, $\operatorname{FMD}(p=.033)$ remained a significant predictor although the relationship between IMT and executive functioning was attenuated ( $p=.11)$. Similarly, after controlling for CVRFs and IMT, GTN-D significantly improved the fit of the executive functioning model (adjusted $\mathrm{R}^{2}$ change $=2.0 \% ; \mathrm{b}=1.52 ; p=.047$ ), but was not significantly related to working memory $(b=0.93 ; p=.294)$. 
CVRFs did not improve the fit of the verbal recall model. Similarly, neither IMT $(b=0.16$; $p=.414)$ nor FMD $(\mathrm{b}=0.33 ; p=.273)$ was associated with the verbal recall composite score in the presence of CVRFs.

\section{DISCUSSION}

Results of this investigation indicate that higher levels of cerebrovascular risk are associated with poorer neuropsychological functioning among adults with MDD. We found that greater levels of CVRFs, including diabetes, hypertension, and smoking, were associated with poorer performance on measures of executive functioning and working memory. Higher levels of HDL cholesterol, in contrast, were actually associated with better executive functioning. Three noninvasive measures of presymptomatic vascular dysfunction-IMT, FMD, and GTN-D—were also associated with decrements in neuropsychological performance. Increasing levels of IMT, an index of subclinical atherosclerosis, were associated with poorer executive functioning but were not associated with working memory after controlling for CVRFs. Poorer endothelial function, indexed by lower FMD, was associated with poorer executive functioning and working memory after controlling for both CVRFs and IMT. Similarly, poorer overall vascular response, indexed by lower GTN-D, was associated with poorer executive functioning after controlling for CVRFs and IMT, although GTN-D was not significantly related to working memory.

Previous population studies have shown that greater levels of CVRFs may be associated with a poorer cognitive functioning (44). In a sample of approximately 11,000 individuals from the Atherosclerotic Risk in Communities study, Knopman and colleagues found that the presence of diabetes and hypertension at baseline testing were associated with more rapid cognitive decline (44). Similarly, Elias and colleagues (3) found that both hypertension and diabetes were associated with poorer neuropsychological performance on a variety of measures, most notably memory. Furthermore, Elias and colleagues (5) found that CVRFs, measured using the FSRP, were associated with poorer performance across a range of neuropsychological tests, including measures of visual-spatial and executive functioning. More recently, Sheline and colleagues (28) found that higher levels of CVRFs were associated with poorer processing speed, memory, and executive functioning in a study of individuals with late-onset depression.

Neuropsychological deficits $(14,15)$ and CVRFs $(9-13)$ are common among individuals with MDD and are associated with cognitive impairment in the general population $(45,46)$. Individuals with MDD have been shown to exhibit neuropsychological deficits over a broad range of cognitive domains (47-49), particularly on tasks associated with frontal lobe activity, such as executive functioning $(9,10)$. Deficits in executive functioning have also been demonstrated among individuals with vascular depression, a late-onset subtype of MDD that is particularly resistant to conventional therapeutic techniques $(9,28)$ and is associated with ischemic damage to the prefrontal-subcortical loops that control executive functions $(50,51)$.

The existing literature on the relationship between serum cholesterol and neuropsychological performance has yielded mixed results. Elias and colleagues found that higher levels of total cholesterol were associated with better neuropsychological performance in the Framingham Heart Study (52). Interestingly, Muldoon and colleagues also reported a positive relationship between total cholesterol and fluid intelligence levels, whereas higher cholesterol was associated with poorer scores on measures of crystallized intelligence (4). Generally, previous studies among older individuals have noted a protective effect of elevated HDL level (53), although the relationship between LDL and cognitive functioning is less clear $(52,54)$. Discrepant findings may result, in part, from the dual role 
of cholesterol as a risk factor for atherosclerosis as well as an agent for delivering nutrients necessary for cognitive functioning (55). Interestingly, several previous studies have reported lower levels of serum cholesterol among individuals with depression (56-61), although not all studies have supported this finding (62).

Our observation that higher IMT is associated with poorer neuropsychological functioning is consistent with previous studies demonstrating an association between greater IMT and lower performance on tests associated with executive functioning $(22,23)$. Auperin and colleagues (23) found that higher levels of IMT measured from the common carotid artery were associated with lower scores on the DSST and COWAT among men, whereas no consistent relationship was noted in women. Mathiesen and colleagues (22) also found that common carotid artery stenosis was associated with poorer scores on a range of neuropsychological tests including the DST, Verbal Paired Associates immediate recall, and Trail Making Tests, although results were strongest for TMT-B. Notably, these associations were not significantly attenuated after controlling for white matter hyperintensities measured by magnetic resonance imaging. In contrast, Knopman and colleagues (44) found that, although cognitive decline was associated with greater CVRFs, carotid IMT was not associated with more rapid decline in neuropsychological performance. Several previous pharmacological interventions have also reported an association between improved vascular functioning and increases in overall cognitive performance $(63,64)$. Furthermore, Muller and colleagues (65) recently reported that higher pulse-wave velocity was associated with poorer performance on cognitive tests assessing processing speed and executive functioning in a sample of middle-aged and older men.

Results from our study indicate that subclinical levels of atherosclerosis, as measured by IMT, FMD, and GTN-D, may be associated with increased neuropsychological deficits. The finding that FMD and GTN-D were significantly associated with executive functioning after controlling for IMT is not surprising, given that IMT is a structural manifestation of systemic vascular disease, whereas endothelial and smooth muscle dysfunction precede the development of IMT (66) and are potentially more sensitive indexes of early atherosclerotic disease (67). The attenuation of the IMT and executive functioning relationship after controlling for FMD is therefore understandable, given the shared variance between these measures and the higher relative sensitivity of FMD. Furthermore, because impaired vascular function, assessed by FMD and GTN-D, may serve as early markers of a generalized vascular burden that precedes the development of manifest atherosclerotic disease, our findings may have important implications for understanding the relationship of subclinical vascular disease and neuropsychological performance.

The finding that CVRFs are associated with reduced performance on tasks of executive functioning may be particularly important among individuals with MDD because poorer executive performance has been associated with an increased risk of relapse among individuals with vascular depression as well as MDD (68). Previous studies have reported that executive functioning is the only domain of cognitive functioning shown to differentiate depressed individuals in remission from healthy controls (69) and has been associated with nonresponsiveness to pharmacologic treatment in patients with MDD.

Deficits in executive functioning may be the result of damage to the frontal-subcortical circuits of the brain (14), a series of discrete pathways supporting executive cognitive functions (70). This region is particularly vulnerable to ischemic injury due to its relatively long, small-diameter, penetrating branches deriving from the anterior and middle cerebral arteries (14). Damage in this region could result in multiple deficits in executive functions because multiple areas of the frontal cortex are perfused by the same "watershed" arteries, thereby conferring a greater risk of microvascular injury during a state of hypoperfusion. 
Because ischemic damage to this area has been associated with the development of vascular depression (21), this may have important implications for individuals with MDD. Furthermore, dysregulation between the prefrontal cortex and the limbic system has been implicated in the pathogenesis of MDD (71) such that damage to this area might be associated with decreased regulatory functioning.

This study has several limitations. First, because we used a cross-sectional design, we are unable to determine if there is a causal relation between CVRFs and neuropsychological deficits. Second, we used a questionnaire to quantify CVRFs and relied on patient selfreports to document the presence of coronary heart disease (CHD) and diabetes. It is possible that other individuals with occult CHD or diabetes might have gone unidentified in our analysis. Third, the possibility exists that the association between MDD and impaired neuropsychological performance is due to a common pathway; for example, both may be a result of hypothalamic-pituitary-adrenal axis dysregulation, which has been associated with both MDD and neuropsychological deficits (72). Finally, due to the limited range of depressive symptoms resulting from our inclusion criteria, it is unclear how depression severity may affect the relationship between neuropsychological performance, CVRFs, and vascular health among individuals with subclinical levels of depression.

In summary, higher levels of CVRFs were associated with deficits in neuropsychological functioning among individuals with MDD. Moreover, impaired vascular function predicted deficits in neurocognitive performance over and above the contribution of higher CVRFs. Future studies should investigate prospectively the relationship between CVRF, vascular functioning, and neuropsychological outcomes among individuals with MDD to determine the natural history of age-related declines in neurocognitive functioning, particularly in the context of impaired vascular function and CVRFs. Furthermore, the extent to which neurocognitive function may be improved post MDD treatment needs further study.

\section{Acknowledgments}

This study was completed in partial fulfillment of the first author's master's thesis. The research was supported by Grants MH 49679 (J.A.B., M.A.B., B.M.H., P.M.D., R.W., A.H., A.S.) and HL080664-01A1 (J.A.B., M.A.B., B.M.H., P.M.D., R.W., A.H., A.S.) from the National Institutes of Health and M01-RR-30 (J.A.B., M.A.B., B.M.H., P.M.D., R.W., A.H., A.S.) from the General Clinical Research Center Program, National Center for Research Resources, and National Institutes of Health.

\section{Glossary}

$\begin{array}{ll}\text { CVRF } & \text { cerebrovascular risk factor } \\ \text { FSRP } & \text { Framingham Stroke Risk Profile } \\ \text { MDD } & \text { major depressive disorder } \\ \text { IMT } & \text { intima medial thickness } \\ \text { GTN } & \text { glyceryl trinitrate } \\ \text { GTN-D } & \text { glyceryl trinitrate dilation } \\ \text { FMD } & \text { flow mediated dilation } \\ \text { HDL } & \text { high-density lipoprotein } \\ \text { LDL } & \text { low-density lipoprotein } \\ \text { Ham-D } & \text { Hamilton Rating Scale for Depression } \\ \text { DSST } & \text { digit symbol substitution test }\end{array}$




$\begin{array}{ll}\text { COWAT } & \text { controlled oral word association test } \\ \text { DST } & \text { digit span test } \\ \text { CHD } & \text { coronary heart disease }\end{array}$

\section{References}

1. Williams GR, Jiang JG, Matchar DB, Samsa GP. Incidence and occurrence of total (first-ever and recurrent) stroke. Stroke. 1999; 30:2523-8. [PubMed: 10582972]

2. Callahan CM, Hendrie HC, Tierney WM. Documentation and evaluation of cognitive impairment in elderly primary-care patients. Ann Intern Med. 1995; 122:422-9. [PubMed: 7856990]

3. Elias PK, Elias MF, Dagostino RB, Cupples LA, Wilson PW, Silbershatz H, Wolf PA. NIDDM and blood pressure as risk factors for poor cognitive performance- the Framingham study. Diabetes Care. 1997; 20:1388-95. [PubMed: 9283785]

4. Muldoon MF, Ryan CM, Matthews KA, Manuck SB. Serum cholesterol and intellectual performance. Psychosom Med. 1997; 59:382-7. [PubMed: 9251158]

5. Elias MF, Sullivan LM, D’Agostino RB, Elias PK, Beiser A, Au R, Seshadri S, DeCarli C, Wolf PA. Framingham stroke risk profile and lowered cognitive performance. Stroke. 2004; 35:404-9. [PubMed: 14726556]

6. Elkins JS, Knopman DS, Yaffe K, Johnston SC. Cognitive function predicts first-time stroke and heart disease. Neurology. 2005; 64:1750-5. [PubMed: 15911803]

7. Geschwind DH, Robidoux J, Alarcon M, Miller BL, Wilhelmsen KC, Cummings JL, Nasreddine ZS. Dementia and neurodevelopmental predisposition: cognitive dysfunction in presymptomatic subjects precedes dementia by decades in frontotemporal dementia. Ann Neurol. 2001; 50:741-6. [PubMed: 11761471]

8. Jonas BS, Mussolino ME. Symptoms of depression as a prospective risk factor for stroke. Psychosom Med. 2000; 62:463-71. [PubMed: 10949089]

9. Austin MP, Mitchell P, Goodwin GM. Cognitive deficits in depression-possible implications for functional neuropathology. Br J Psychiatry. 2001; 178:200-6. [PubMed: 11230029]

10. Austin MP, Mitchell P, Wilhelm K, Parker G, Hickie I, Brodaty H, Chan J, Eyers K, Milic M, Hadzi-Pavlovic D. Cognitive function in depression: a distinct pattern of frontal impairment in melancholia? Psychol Med. 1999; 29:73-85. [PubMed: 10077295]

11. Yochim BP, Kerkar SP, Lichtenberg PA. Cerebrovascular risk factors, activity limitations, and depressed mood in African American older adults. Psychol Aging. 2006; 21:186-9. [PubMed: 16594803]

12. Thomas AJ, Kalaria RN, O'Brien JT. Depression and vascular disease: what is the relationship? J Affect Disord. 2004; 79:81-95. [PubMed: 15023483]

13. Mast BT, Neufeld S, MacNeill SE, Lichtenberg PA. Longitudinal support for the relationship between vascular risk factors and late-life depressive symptoms. Am J Geriatr Psychiatry. 2004; 12:93-101. [PubMed: 14729564]

14. Campbell JJ, Coffey CE. Neuropsychiatric significance of subcortical hyperintensity. J Neuropsychiatry Clin Neurosci. 2001; 13:261-88. [PubMed: 11449035]

15. Mega MS, Cummings JL. Frontal-subcortical circuits and neuropsychiatric disorders. J Neuropsychiatry Clin Neurosci. 1994; 6:358-70. [PubMed: 7841807]

16. Barnes DE, Alexopoulos GS, Lopez OL, Williamson JD, Yaffe K. Depressive symptoms, vascular disease, and mild cognitive impairment-findings from the cardiovascular health study. Arch Gen Psychiatry. 2006; 63:273-80. [PubMed: 16520432]

17. Janssen J, Pol HEH, Lampe IK, Schnack HG, de Leeuw FE, Kahn RS, Heeren TJ. Hippocampal changes and white matter lesions in early-onset depression. Biol Psychiatry. 2004; 56:825-31. [PubMed: 15576058]

18. Steffens DC, Helms MJ, Krishnan KRR, Burke GL. Cerebrovascular disease and depression symptoms in the cardiovascular health study. Stroke. 1999; 30:2159-66. [PubMed: 10512922] 
19. Murphy CF, Gunning-Dixon F, Hoptman MJ, Ardekan BA, Shields JK, Lim KO, Alexopoulos GS. White matter integrity predicts stroop performance in patients with geriatric depression. Biol Psychiatry. 2005; 57:30S-1S.

20. Heiden A, Kettenbach K, Fischer P, Schein B, Ba-Ssalamah A, Frey R, Gulesserian T, Itzlinger U, Trattnig S, Imhof H, Kasper S. White matter lesions and chronicity of depression. Eur Psychiatry. 2000; 15:368S.

21. Alexopoulos GS, Meyers BS, Young RC, Campbell S, Silbersweig D, Charlson M. "Vascular depression" hypothesis. Arch Gen Psychiatry. 1997; 54:915-22. [PubMed: 9337771]

22. Mathiesen EB, Waterloo K, Joakimsen O, Bakke SJ, Jacobsen EA, Bonaa KH. Reduced neuropsychological test performance in asymptomatic carotid stenosis-the Tromso study. Neurology. 2004; 62:695-701. [PubMed: 15007116]

23. Auperin A, Berr C, BonithonKopp C, Touboul PJ, Ruelland I, Ducimetiere P, Alperovitch A. Ultrasonographic assessment of carotid wall characteristics and cognitive functions in a community sample of 59- to 71-year-olds. Stroke. 1996; 27:1290-5. [PubMed: 8711788]

24. Elovainio M, Keltikangas-Jarvinen L, Kivimaki M, Pulkki L, Puttonen S, Heponiemi T, Juonala M, Viikari JSA, Raitakari OT. Depressive symptoms and carotid artery intima-media thickness in young adults: the cardiovascular risk in young Finns study. Psychosom Med. 2005; 67:561-7. [PubMed: 16046368]

25. Sherwood A, Hinderliter AL, Watkins LL, Waugh RA, Blumenthal JA. Impaired endothelial function in coronary heart disease patients with depressive symptomatology. J Am Coll Cardiol. 2005; 46:656-9. [PubMed: 16098431]

26. Touboul PJ, Labreuche J, Vicaut E, Amarenco P. Carotid intima-media thickness, plaques, and Framingham risk score as independent determinants of stroke risk. Stroke. 2005; 36:1741-5. [PubMed: 16020769]

27. Kannel WB, Vasan RS, Levy D. Is the relation of systolic blood pressure to risk of cardiovascular disease continuous and graded, or are there critical values? Hypertension. 2003; 42:453-6. [PubMed: 12975387]

28. Sheline YI, Barch DM, Garcia K, Gersing K, Pieper C, Welsh-Bohmer K, Steffens DC, Doraiswamy PM. Cognitive function in late life depression: Relationships to depression severity, cerebrovascular risk factors and processing speed. Biol Psychiatry. 2006; 60:58-65. [PubMed: 16414031]

29. Jones DJ, Bromberger JT, Sutton-Tyrrell K, Matthews KA. Lifetime history of depression and carotid atherosclerosis in middle-aged women. Arch Gen Psychiatry. 2003; 60:153-60. [PubMed: 12578432]

30. American Psychiatric Association. Diagnostic and statistical manual of mental disorders-IVtext revision. 4. Washington, DC: American Psychiatric Association; 2004.

31. D’Agostino RB, Wolf PA, Belanger AJ, Kannel WB. Stroke risk profile: adjustment for antihypertensive medication. The Framingham study. Stroke. 1994; 25:40-3. [PubMed: 8266381]

32. Wolf PA, D' Agostino RB, Belanger AJ, Kannel WB. Probability of stroke: a risk profile from the Framingham Study. Stroke. 1991; 22:312-8. [PubMed: 2003301]

33. Wendelhag I, Gustavsson T, Suurküla M, Berglund G, Wikstrand J. Ultrasound measurement of wall thickness in the carotid artery: fundamental principles and description of a computerized analysing system. Clin Physiol. 1991; 11:565-77. [PubMed: 1769190]

34. Wechsler, D. Wechsler memory scale—revised. New York: Psychological Corp; 1987.

35. Spreen, O.; Strauss, E. A compendium of neuropsychological tests. New York: Oxford University Press; 1991.

36. Wechsler, D. Manual for the Wechsler adult intelligence scale. New York: Psychological Corporation; 1956.

37. Ruff RM, Niemann H, Allen CC. The Ruff 2 and 7 selective attention test: a neuropsychological application. Percept Mot Skills. 1992; 75:1311-9. [PubMed: 1484803]

38. Stroop JR. Studies of interference in serial verbal reactions. J Exp Psychiat. 1935; 18:643-62.

39. Lezak, MD. Neuropsychological assessment. 3. New York: Oxford University Press; 1995.

40. Reitan, RM. Manual for administration of neuropsychological test batteries for adults and children. Tucson: Reitan Neuropsychological Laboratories, Inc; 1979. 
41. Robins LN, Helzer JE, Croughan J, Ratcliff KS. National Institute of Mental health diagnostic interview schedule. Its history, characteristics, and validity. Arch Gen Psychiatry. 1981; 38:381-9. [PubMed: 6260053]

42. American Psychological Association. Diagnostic and statistical manual of mental disorders. Washington, DC: American Psychological Association; 1994.

43. Williams JBW. A structured interview guide for the Hamilton depression rating scale. Arch Gen Psychiatry. 1988; 45:742-7. [PubMed: 3395203]

44. Knopman D, Boland LL, Mosley T, Howard G, Liao D, Szklo M, McGovern P, Folsom AR. Cardiovascular risk factors and cognitive decline in middle-aged adults. Neurology. 2001; 56:428. [PubMed: 11148234]

45. Breteler MMB. Vascular risk factors for Alzheimer's disease: an epidemiologic perspective. Neurobiol Aging. 2000; 21:153-60. [PubMed: 10867200]

46. Birkenhager WH, Forette F, Seux ML, Wang JG, Staessen JA. Blood pressure, cognitive functions, and prevention of dementias in older patients with hypertension. Arch Intern Med. 2001; 161:1526. [PubMed: 11176727]

47. Porter RJ, Gallagher P, Thompson JM, Young AH. Neurocognitive impairment in drug-free patients with major depressive disorder. Br J Psychiatry. 2003; 182:214-20. [PubMed: 12611784]

48. Elderkin-Thompson V, Kumar A, Bilker WB, Dunkin JJ, Mintz J, Moberg PJ, Mesholam RI, Gur RE. Neuropsychological deficits among patients with late-onset minor and major depression. Arch Clin Neuropsychol. 2003; 18:529-49. [PubMed: 14591448]

49. Rund BR, Sundet K, Asbjornsen A, Egeland J, Landro NI, Lund A, Roness A, Stordal KI, Hugdahl K. Neuropsychological test profiles in schizophrenia and non-psychotic depression. Acta Psychiatr Scand. 2006; 113:350-9. [PubMed: 16638080]

50. Oh BH, Cheon JS. White matter lesions on magnetic resonance imaging in late-life depression. Psychogeriatrics. 2004; 4:72-81.

51. Tullberg M, Fletcher E, DeCarli C, Mungas D, Reed BR, Harvey DJ, Weiner MW, Chui HC, Jagust WJ. White matter lesions impair frontal lobe function regardless of their location. Neurology. 2004; 63:246-53. [PubMed: 15277616]

52. Elias PK, Elias MF, D’Agostino RB, Sullivan LM, Wolf PA. Serum cholesterol and cognitive performance in the Framingham heart study. Psychosom Med. 2005; 67:24-30. [PubMed: 15673620]

53. Atzmon G, Gabriely I, Greiner W, Davidson D, Schechter C, Barzilai N. Plasma HDL levels highly correlate with cognitive function in exceptional longevity. J Gerontol A Biol Sci Med Sci. 2002; 57:M712-M715. [PubMed: 12403798]

54. Yaffe K, Barrett-Connor E, Lin F, Grady D. Serum lipoprotein levels, statin use, and cognitive function in older women. Arch Neurol. 2002; 59:378-84. [PubMed: 11890840]

55. Muldoon, MF.; Flory, JD.; Ryan, CM. Serum cholesterol, the brain, and cognitive functioning. In: Waldstein, SR.; Elias, MF., editors. Neuropsychology of cardiovascular disease. London: Lawrence Erlbaum Associates; 2001.

56. Maes M, Smith R, Christophe A, Vandoolaeghe E, Van GA, Neels H, Demedts P, Wauters A, Meltzer HY. Lower serum high-density lipoprotein cholesterol (HDL-C) in major depression and in depressed men with serious suicidal attempts: relationship with immune-inflammatory markers. Acta Psychiatr Scand. 1997; 95:212-21. [PubMed: 9111854]

57. Rozzini R, Bertozzi B, Barbisoni P, Trabucchi M. Low serum cholesterol and serotonin metabolism. Risk of depression is higher in elderly patients with lowest serum cholesterol values. BMJ. 1996; 312:1298-9. [PubMed: 8634630]

58. Brown SL, Salive ME, Harris TB, Simonsick EM, Guralnik JM, Kohout FJ. Low cholesterol concentrations and severe depressive symptoms in elderly people. BMJ. 1994; 308:1328-32. [PubMed: 8019218]

59. Dursun SM, Burke JG, Reveley MA. Low serum cholesterol and depression. BMJ. 1994; 309:2734. [PubMed: 8069163]

60. Partonen T, Haukka J, Virtamo J, Taylor PR, Lonnqvist J. Association of low serum total cholesterol with major depression and suicide. Br J Psychiatry. 1999; 175:259-62. [PubMed: 10645328] 
61. Maes M, Smith R, Christophe A, Vandoolaeghe E, Van GA, Neels H, Demedts P, Wauters A, Meltzer HY. Lower serum high-density lipoprotein cholesterol (HDL-C) in major depression and in depressed men with serious suicidal attempts: relationship with immune-inflammatory markers. Acta Psychiatr Scand. 1997; 95:212-21. [PubMed: 9111854]

62. McCallum J, Simons L, Simons J, Friedlander Y. Low serum cholesterol is not associated with depression in the elderly: data from an Australian community study. Aust N Z J Med. 1994; 24:561-4. [PubMed: 7848160]

63. Moser DJ, Hoth KF, Robinson RG, Paulsen JS, Sinkey CA, Benjamin ML, Schultz SK, Haynes WG. Blood vessel function and cognition in elderly patients with atherosclerosis. Stroke. 2004; 35:E369-E372. [PubMed: 15472091]

64. Hanon O, Haulon S, Lenoir H, Seux ML, Rigaud AS, Safar M, Girerd X, Forette F. Relationship between arterial stiffness and cognitive function in elderly subjects with complaints of memory loss. Stroke. 2005; 36:2193-7. [PubMed: 16151027]

65. Muller M, Grobbee DE, Aleman A, Bots M, van der Schouw YT. Cardiovascular disease and cognitive performance in middle-aged and elderly men. Atherosclerosis. 2007; 190:143-9. [PubMed: 16488420]

66. Celermajer DS, Sorensen KE, Gooch VM, Spiegelhalter DJ, Miller OI, Sullivan ID, Lloyd JK, Deanfield JE. Noninvasive detection of endothelial dysfunction in children and adults at risk of atherosclerosis. Lancet. 1992; 340:1111-5. [PubMed: 1359209]

67. Reddy KG, Nair RN, Sheehan HM, Hodgson JM. Evidence that selective endothelial dysfunction may occur in the absence of angiographic or ultrasound atherosclerosis in patients with risk-factors for atherosclerosis. J Am Coll Cardiol. 1994; 23:833-43. [PubMed: 8106687]

68. Majer M, Ising M, Kunzel H, Binder EB, Holsboer F, Modell S, Zihl J. Impaired divided attention predicts delayed response and risk to relapse in subjects with depressive disorders. Psychol Med. 2004; 34:1453-63. [PubMed: 15724876]

69. Paelecke-Habermann Y, Pohl J, Leplow B. Attention and executive functions in remitted major depression patients. J Affect Disord. 2005; 89:125-35. [PubMed: 16324752]

70. Mega MS, Cummings JL. Frontal-subcortical circuits and neuropsychiatric disorders. J Neuropsychiatry Clin Neurosci. 1994; 6:358-70. [PubMed: 7841807]

71. Davidson RJ, Pizzagalli D, Nitschke JB, Putnam K. Depression: Perspectives from affective neuroscience. Annu Rev Psychol. 2002; 53:545-74. [PubMed: 11752496]

72. Allister-Williams RH, Ferrier IN, Young AH. Mood and neuropsychological function in depression: the role of corticosteroids and serotonin. Psychol Med. 1998; 28:573-84. [PubMed: 9626714] 


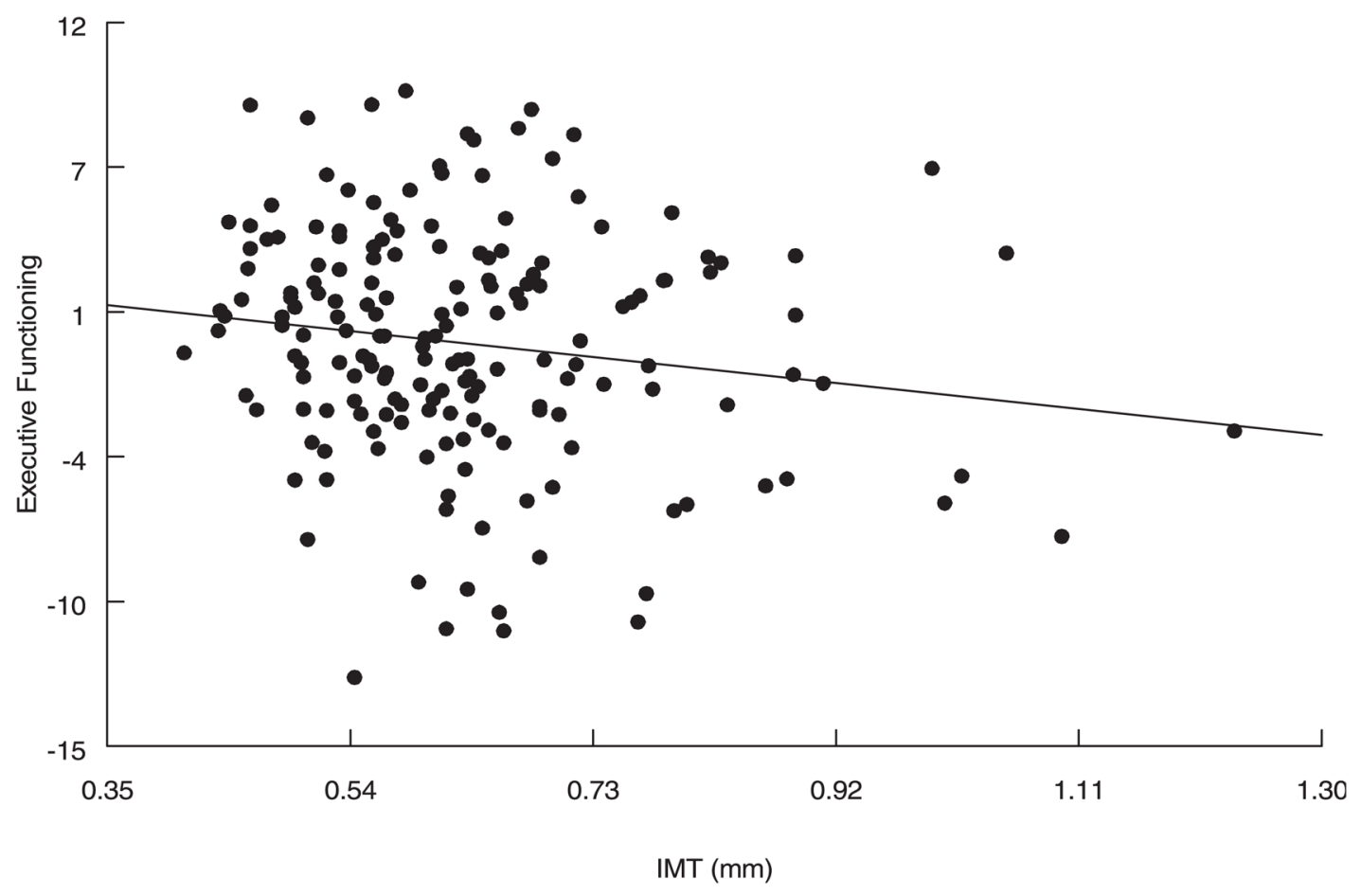

Figure 1.

Intima medial thickness (IMT) and executive functioning. Adjusted for age, education, depression, serum cholesterol, and Framingham Stroke Risk Profile levels. 


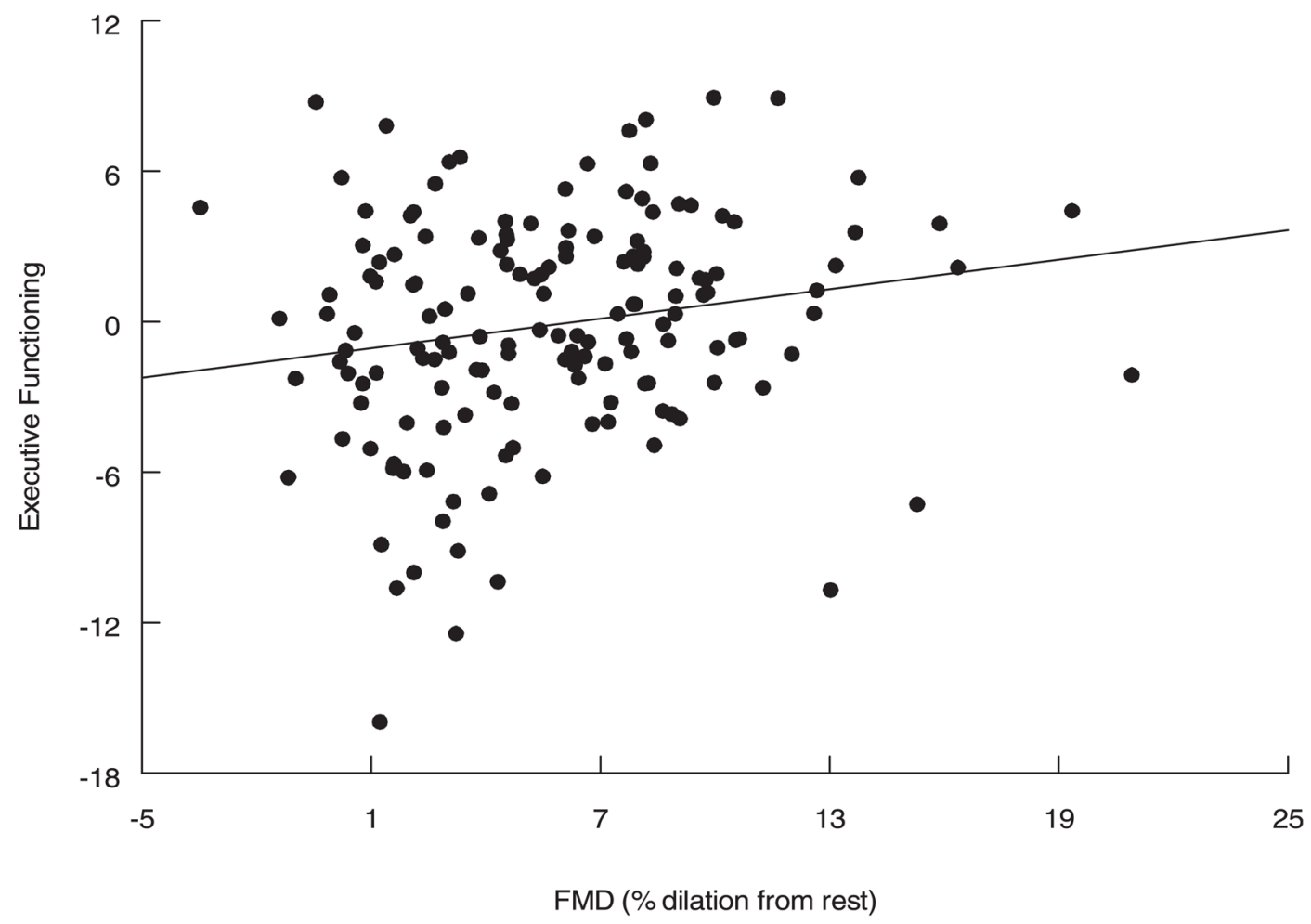

Figure 2.

Flow mediated dilation (FMD) and executive functioning. Adjusted for age, education, depression, serum cholesterol, and Framingham Stroke Risk Profile levels. 


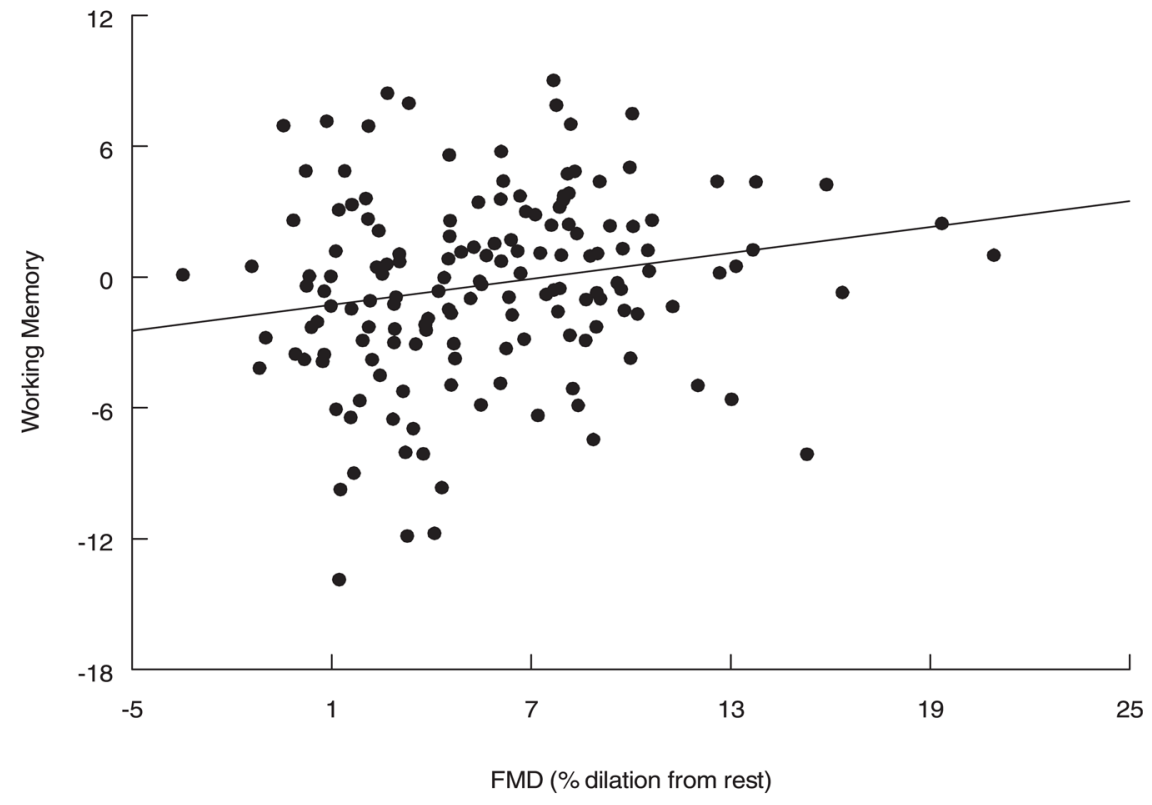

Figure 3.

Flow mediated dilation (FMD) and working memory. Adjusted for age, education, depression, serum cholesterol, and Framingham Stroke Risk Profile levels. 


\section{TABLE 1}

\section{Background Characteristics}

\begin{tabular}{lcc}
\hline Variable & Mean & SD \\
\hline Age, mean, years & 51.6 & 7.5 \\
Males, n (\%) & $48(24)$ & \\
Whites, n (\%) & $134(68)$ & \\
College graduate, n (\%) & $100(51)$ & \\
Ham-D, mean & 17 & 4.3 \\
Married, n (\%) & 75 & 49 \\
IMT, mean, mm & 0.63 & 0.13 \\
GTN-D, \% & 20.7 & 8.23 \\
FMD, \% & 5.76 & 4.13 \\
Current smoker, n (\%) & $32(16)$ & \\
Previous smoker (excluding current use), $\mathrm{n}(\%)$ & $65(38)$ & \\
SBP, mean, mm Hg & 124 & 18 \\
DBP, mean, mm Hg & 79 & 10 \\
BP-lowering medications, $\mathrm{n}(\%)$ & $47(23)$ & \\
Total cholesterol, mean, mg/dl & 207 & 41 \\
LDL total, mean, mg/dl & 122 & 33 \\
HDL total mean, mg/dl & 56.9 & 15.8 \\
Lipid-lowering medications, $\mathrm{n}(\%)$ & $17(8)$ & \\
BMI, kg/m ${ }^{2}$ & 30.1 & 7.1 \\
Framingham Risk Profile Score & 5.4 & 3.2 \\
\hline
\end{tabular}

Data are given as number (percentage) unless otherwise indicated.

$\mathrm{SD}=$ standard deviation; Ham-D = Hamilton Rating Scale for Depression; IMT = intima medial thickness; GTN-D = glyceryl trinitrate dilation; $\mathrm{FMD}=$ flow mediated dilation; $\mathrm{SBP}=$ systolic blood pressure DBP = diastolic blood pressure $; \mathrm{BP}=$ blood pressure $; \mathrm{LDL}=$ low-density lipoprotein; HDL = high-density lipoprotein; BMI = body mass index. 
TABLE 2

Neuropsychological Test Scores

\begin{tabular}{lrr}
\hline Test & Mean & SD \\
\hline Ruff 2 and 7 test & 231.9 & 49.5 \\
Digit symbol substitution test & 57.6 & 11.5 \\
Stroop color/word & 36.5 & 9.8 \\
Stroop color & 68.7 & 12.3 \\
Stroop word & 95.2 & 14.8 \\
Trail's A time (sec) & 28.1 & 10.1 \\
Trail's B time (sec) & 70.3 & 35.8 \\
Logical memory & 24.7 & 6.5 \\
Verbal recall easy & 11.0 & 1.2 \\
Verbal recall hard & 7.1 & 2.7 \\
DST forward & 8.3 & 2.3 \\
DST backward & 6.6 & 2.1 \\
COWAT & 39.5 & 11.9 \\
Animal naming & 19.4 & 4.9 \\
\hline
\end{tabular}

$\mathrm{SD}=$ standard deviation $; \mathrm{DST}=$ digit span test COWAT $=$ controlled oral word association test . 
TABLE 3

Factor Loadings With Individual Tests ${ }^{a}$

\begin{tabular}{lrrr}
\hline Test & Executive Functioning & Verbal Recall & Working Memory \\
\hline Ruff total & 0.79 & 0.07 & 0.17 \\
Digit symbol & 0.82 & 0.32 & 0.32 \\
Stroop color/word & 0.66 & 0.08 & 0.54 \\
Stroop color & 0.77 & -0.07 & 0.52 \\
Stroop word & 0.58 & -0.14 & 0.60 \\
Trail's A & -0.63 & -0.07 & -0.14 \\
Trail's B & -0.78 & -0.16 & -0.43 \\
Logical memory & 0.16 & 0.66 & -0.01 \\
Verbal recall easy & 0.02 & 0.75 & -0.01 \\
Verbal recall hard & 0.08 & 0.83 & 0.13 \\
Digit forward & 0.32 & -0.01 & 0.74 \\
Digit backward & 0.37 & 0.18 & 0.74 \\
COWAT & 0.27 & 0.15 & 0.56 \\
Animal naming & 0.28 & 0.30 & 0.40 \\
\hline
\end{tabular}

COWAT $=$ controlled oral word association test

${ }^{a}$ A minimum loading of 0.40 was required to be retained in the composite variable. 


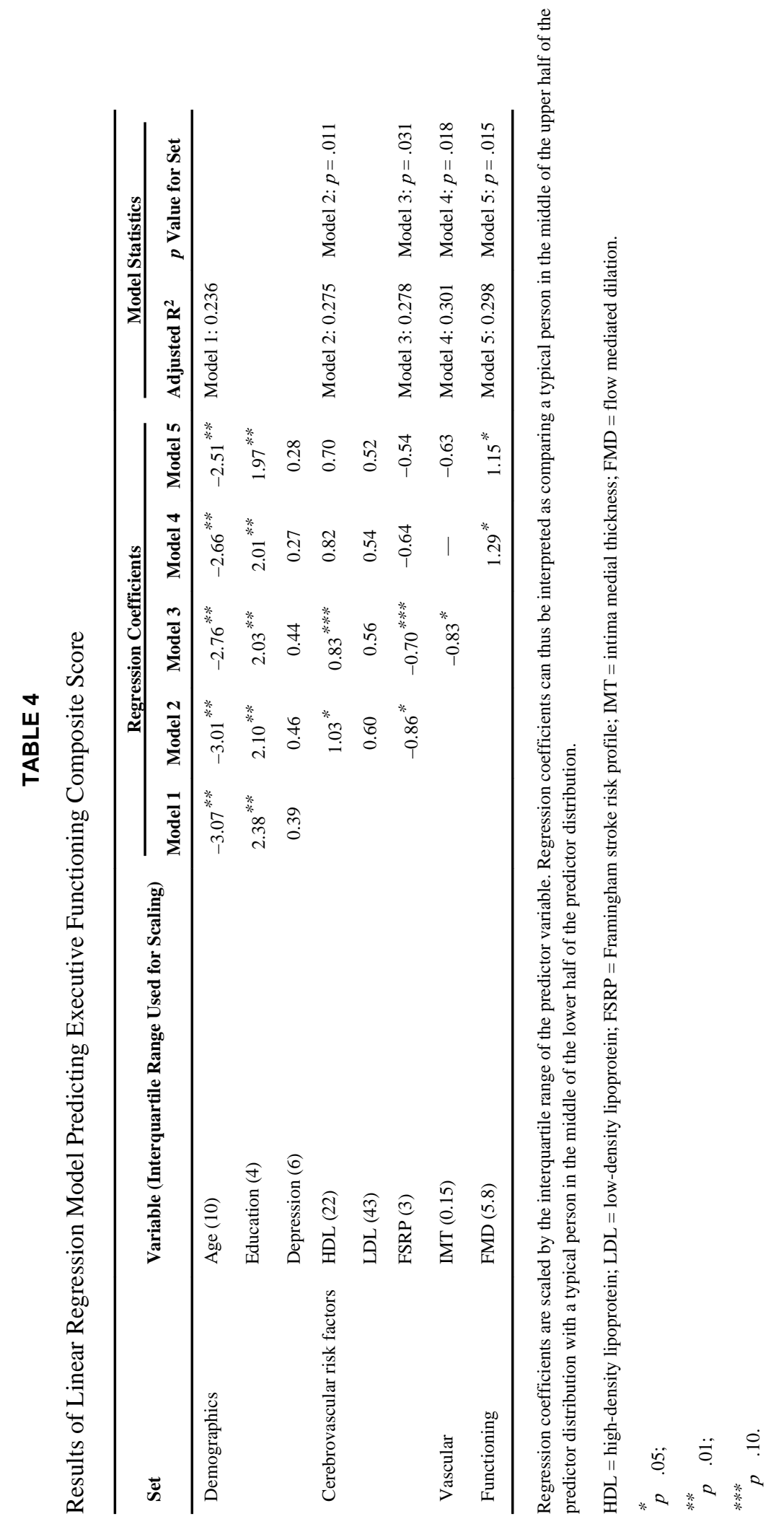

Psychosom Med. Author manuscript; available in PMC 2013 March 13. 


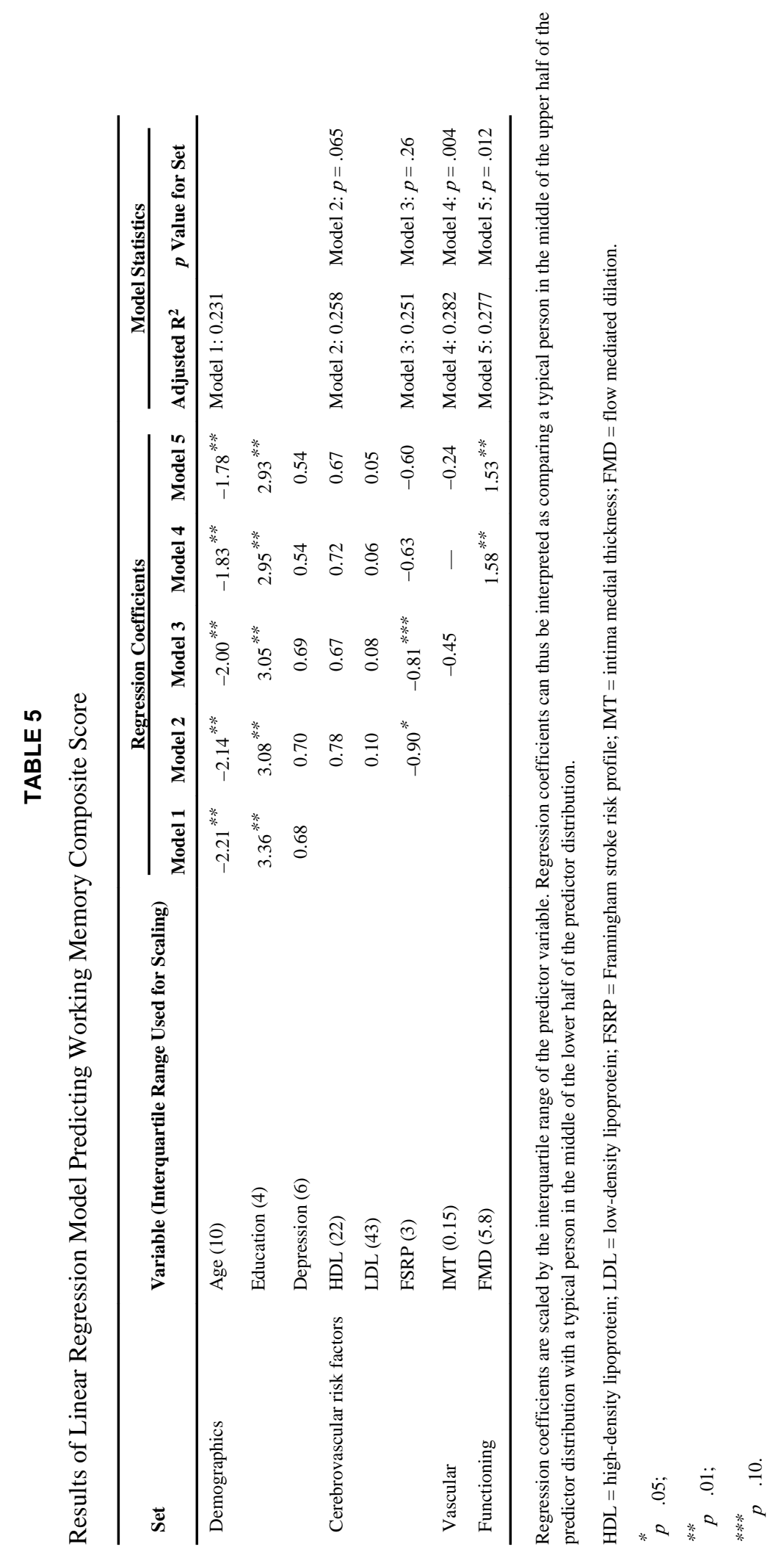

Psychosom Med. Author manuscript; available in PMC 2013 March 13. 